\title{
Expanded role of one stage indications in total knee arthroplasty revision
}

Keywords: Single stage, one stage, direct exchange, prosthetic joint infection, total knee arthroplasty

\section{Background}

The utilization of total knees has a projected increase to 3.48 million cases by 2030 . Revisions are also projected to increase $601 \%$ from $2005-2030$ to 268,200 cases, $65.5 \%$ of which are projected to be revisions related to infection. ${ }^{1}$ In 1983 John Insall MD described a two stage revision approach as a way to salvage an infected total knee arthroplasty (TKA) with 11 knees and no recurrences. It was noted that the method was both "costly and time-consuming." Despite cost and time consumption, a two stage has become and remained the gold standard of TKA infection management. ${ }^{3}$ Alternatives for infection management are limited to irrigation and debridement, resection arthroplasty, arthrodesis, amputation, chronic suppression and one stage revision arthroplasty., ${ }^{4,5}$ Two stage revision failure rates have been variable between $0-41 \%{ }^{6}$ with average failure rates being found to be between $17.3-18 \% .{ }^{7.8}$ In addition to risks for reduced mobility, recurrent infection, stiffness/contracture, instability and spacer fracture, ${ }^{9}$ two studies regarding two stage revision arthroplasties have been published showing significantly elevated mortality rates at 90days of $1.7-4 \%$, up to $16 \%$ at 5 years and $22 \%$ overall. ${ }^{7,10}$

As an alternative, single stage management for infected TKA's have been utilized starting back to $1975^{11}$ and more widely adopted in specialized centers in Europe over the years. ${ }^{12}$ One stage arthroplasty leaves the possibility of minimizing stiffness, arthrofibrosis, and cost in comparison to two stage technique., ${ }^{4,13}$ The purpose of this paper is to explore what has been learned from currently published data regarding one stage revisions and to see if the "gold standard" in the United States should be evolving for certain patient populations where we can spare the excess burden of time, resources and potential compromised outcomes that a two stage may lend.

\section{Methods}

A review of the literature on one stage revision arthroplasties was performed and assessed in regards to infection recurrence but also in regards to patient selection, surgical technique, and postoperative antibiotic management, as to better gain an understanding of the potential indications for a one stage arthroplasty revision

\section{Results}

One stage TKA results have ranged in success for infection eradication from $73 \%$ to $100 \%$ (Table 1 ). There has been a trend towards more success with infection eradication in more recently published studies without changes in duration of follow-up..$^{14}$ This can be for a variety of reasons including: a better understanding of bacteria, biofilm, sensitivities, antibiotic options, cement makeup, IV and PO antibiotics and the duration of therapy required and utilization of chemical debridement to supplement mechanical debridement. The key lies in attempting to predict whom a one stage will be most successful in and if two stage revision remains necessary in certain patient populations. The host seems to play

\author{
Volume 10 Issue 3 - 2018
}

\author{
Daniel R Mesko DO,Wayne Paprosky MD \\ Department of Orthopedic Surgery, Rush University Medical \\ Center, USA
}

Correspondence: Daniel R Mesko DO, Midwest Orthopaedics at RUSH, I I6I W Harrison St Chicago, IL 606 I2, USA, Tel (5 I7) 88I-2423, Email dan.mesko@gmail.com

Received: June 06, 2018 | Published: June 15, 2018

a large role in infection, therefore patient selection seems to be fundamental in the success of surgical intervention. Multiple studies have published their recommendations on criteria for who qualifies for one stage intervention in infected TKA. ${ }^{12,15-17}$ Recommendations put forth by the International Society for Infectious disease for one stage arthroplasty included: patients with: no need for bone graft, an aggressive debridement of all infected tissue, satisfactory soft tissue, absence of difficult to treat microorganisms, and a bacteria that is sensitive to antibiotic cement. ${ }^{3}$ There seems to be agreement that an unknown pathogen or a known pathogen of high virulence, acutely decompensated or systemically ill patients, significant soft tissue involvement and general medical contraindications such as immunocompromised states are not optimal candidates for one stage arthroplasty. Silva et al. concluded that 4 important factors contributing to the success of two stage outcomes lies in having a gram positive infection, usage of antibiotic cement with replantation, 12 weeks of postoperative antibiotics and the absence of a sinus tract. ${ }^{5}$

The question lies in how patients perform with strict criteria applied, knowing that around $18 \%$ of patients fail two stage revisions without such criteria, ${ }^{s}$ and then extrapolating, how have one stage revisions performed with less strict inclusion criteria. Utilizing strict patient exclusion criteria, including local factors (skin, soft tissue bony defects/quality, peripheral vascular disease), host factors (immunosuppression, sepsis, reinfection, systemic disease) and organism factors (unknown, resistant or poly bacterial infection) Haddad et al. was able to achieve a $100 \%$ success rate at 36 month average among 28patients. ${ }^{17}$ Singer et al., ${ }^{18}$ did not limit based on host factors, however, excluded MRSA, MRSE, and unknown pathogens as well as wound defects unable to be primarily closed, found a $95 \%$ success rate among 63 patients. ${ }^{18}$ Both of these selective studies certainly faired well in regards to 2 stage success rates in the literature. Interestingly, as many studies suggest soft tissue coverage need, sinus tracts, polymicrobial infections or resistant bacteria are contraindications to a one stage TKA; these also are similar factors that lend to a higher risk of re-infection in two stage revision arthroplasty. ${ }^{6,7}$ Mortazavi et al in their review of two stage arthroplasty studies concluded that culture negative infection and resistant bacteria led to a 4 and 2 fold respectively increased risk of failure. ${ }^{8}$

In regards to how one-stage revisions have fared in a more complex patient population, one of the largest one-stage TKA studies published to date by Zahar et al. ${ }^{15}$ obtained $93 \%$ success in infection 
eradication at 120 month mean follow-up. ${ }^{15}$ Exclusion criteria was limited to negative preoperative aspiration for culture, allergy to cement, and involvement of NV structures. As such, patients were included irrespective of host factors such as: ASA, BMI, sinus tracts or flap requirement. Twenty-one patients were included who had MRSA/MRSE(9), fungal(1), polymicrobial(3), gram negative(8) infections. These success rates were also obtained despite limited post operative antibiotic usage of 14days average without prolonged oral antibiotics. Bauer et al., ${ }^{19}$ also concluded in comparison of 30 single-stage vs 77 two-stage TKAs found that chronicity of infection, number of prior surgeries and bacterial virulence/resistance were not predictive of failure of one surgical technique over another. ${ }^{19}$ Jenny et al. ${ }^{20}$ achieved an $87 \%$ success rate ( $91 \%$ from initial infection) despite including 5patients who required soft tissue flaps and 11 polymicrobial infections..$^{20}$ Fungal infections and repeat failures of 2 prior infections were not included. Three of the 6infections were recurrent, whereas 3 were different bacteria isolated at time of infection and not true recurrences. ${ }^{20}$ There has been one study looking at 4 knees with known fungal infections, which were treated with single stage revision arthroplasty. Three of the 4 were successfully treated as of $7 \mathrm{yrs}$ mean follow up. ${ }^{21}$ Interestingly, $75 \%$ of the fungal infections were also bacterial infected re-enforcing that isolated antifungal therapy may not be appropriate treatment. Ultimately, a more lax selection criteria is not proven definitively in the literature to provide better success rates with two stage over one stage leading to Kunustor et al. to conclude that "one stage TKA's may be as effective as two- stage in treating infected TKA in generally unselected patients. ${ }^{22}$ "

Table I Single stage TKA results

\begin{tabular}{lllll}
\hline & Patient number & Follow up (months) & Success (\%) & Published \\
\hline Freeman et al. ${ }^{11}$ & 8 & 12 to 40 & 100 & 1985 \\
Von Forester et al. ${ }^{28}$ & 104 & 75.5 & 73.1 & 1991 \\
Goksan \& Freeman ${ }^{29}$ & 18 & 60 & 88.8 & 1992 \\
Lu et al. $^{30}$ & 8 & 20.1 & 87.5 & 1997 \\
Buechel et al. ${ }^{31}$ & 22 & 122.4 & 90.9 & 2004 \\
Sofer et al. ${ }^{32}$ & 15 & 18.4 & 93 & 2005 \\
Whiteside $^{24}$ & 18 & 62 & 94.4 & 2011 \\
Parkinson et al. $^{4}$ & 12 & 24 & 100 & 2011 \\
Singer et al. ${ }^{18}$ & 63 & 35.9 & 95 & 2012 \\
Jenny et al. ${ }^{20}$ & 47 & 36 & 87 & 2013 \\
Tibrewal et al. ${ }^{23}$ & 50 & 126 & 98 & 2014 \\
Klatte et al. ${ }^{21}$ & 4 & 84 & 75 & 2014 \\
Haddad et al. ${ }^{17}$ & 28 & 78 & 100 & 2014 \\
Zahar et al. ${ }^{15}$ & 70 & 120 & 93 & 2015 \\
\hline
\end{tabular}

\section{Surgical technique}

Standardizing a surgical technique is another important factor which will lead to more consistently reliable results in terms of infection management. Tibrewal et al. ${ }^{23}$ from the George et al. ${ }^{16}$ in Canada both detail similarity in their papers. ${ }^{16,23}$ Surgical technique at a minimum consists of explant of all components and cement, obtaining tissue for culture from separate sites, radical irrigation and debridement of all involved tissue including posterior capsule, bone etc.. Next, most techniques describe some form of chemical debridement, George et al. utilizing hydrogen peroxide $3 \%$ solution and betadine, ${ }^{16}$ others using polyhexanide ${ }^{21}$ or Octenidine dihydrochloride..$^{18}$ The wound is temporarily closed while it continues to soak in betadine. The contaminated instruments, gowns/gloves and drapes are all changed and the patient is re-prepped for the replant while the surgical team rescrubs. The betadine is irrigated out of the wound and re-implantation ensues with antibiotic cement based on bacterial sensitivities. Antibiotic pellets are placed in the tissue throughout closure. In treating the case as two separate procedures, explant and then replant, the chances of cross contamination as opposed to true recurrence due to ineffective initial debridement would seem to be minimized.

\section{Antibiotic management}

In regards to antibiotics, most facilities defer to infectious disease in regards to selection, duration of treatment with IV, need for oral therapy etc... The International Society for Infectious Disease recommends 2 weeks of IV therapy followed by 6 months of oral therapy for prosthetic knee joint infections. ${ }^{3}$ There was not a general acceptance as to the duration of antibiotic therapy in this pool of international studies as it ranged from as little as 5days IV with conversion to orals, ${ }^{16}$ to $10-17$ days IV without prolonged oral therapy $^{15}$ to intraarticular infusion for 6 weeks. ${ }^{24}$ As mentioned, alternatives to systemic antibiotic therapy have been and continue to be explored ,as Whiteside et al. ${ }^{24}$ described a technique involving single stage knee revision plus Hickman intraarticular infusion of IV vancomycin, which hope to provide elevated antibiotic concentrations 
where they are needed most while minimizing systemic toxicity issues. ${ }^{24}$ This resulted in a $94 \%$ success rate in $17 / 18$ patients. This was again described in 2017 in the hip where 20/21 patients had success eradication with a similar technique. ${ }^{25}$ Most facilities see 4-6weeks IV therapy as a common duration for IV antibiotic therapy in the United States in addition to an antibiotic cement spacer. ${ }^{26}$

\section{Conclusion}

As more evidence is compiled on the management of infection with one stage TKA, there may be an expanding role for its use to minimize infection recurrence while at the same time optimizing patient outcome and improving upon cost and resource utilization. In the two meta-analyses published to date on single stage knee revision, ${ }^{6,14}$ both reiterate that there is a much larger body of literature on two stage revisions and until larger studies of more quality are done on one stage TKA revisions, the gold standard remains two stage by default. A standardized technique for patient inclusion/exclusion, preoperative workup, mechanical and chemical debridement, antibiotic management based on sensitivities, for one stage, remains necessary. At a minimum, knowing the involved pathogen preoperatively should remain the most basic of criteria for one stage TKA revision with factors such as patients ability to undergo further operations, virulence of bacteria involved, medical comorbidities remaining at the discretion of the surgeon and infectious disease specialists, along with patient education on risks and benefits of a one stage vs two stage arthroplasty. Currently a multicenter trial is undergoing active enrollment among tertiary care centers in the United States which will hopefully be able to shed more light on the subject in regards to further standardization among patient selection surgical techniques and post-operative management to optimize management of prosthetic knee infection in a manner that optimizes patient outcomes in as conservative a manner as possible. ${ }^{27}$

\section{Acknowledgements}

None.

\section{Conflict of interest}

Authors declare that there is no conflict of interest.

\section{References}

1. Kurtz S, Ong K, Lau E, et al. Projections of primary and revision hip and knee arthroplasty in the United States from 2005 to 2030. J Bone Joint Surg Am. 2007;89(4):780-785.

2. Insall JN, Thompson FM, Brause BD. Two-stage reimplantation for the salvage of infected total knee arthroplasty. J Bone Joint Surg Am. 1983;65(8):1087-1098.

3. Leone S, Borrè S, Monforte $\mathrm{Ad}$, et al. Consensus document on controversial issues in the diagnosis and treatment of prosthetic joint infections. Int J Infect Dis. 2010;14(Suppl 4):S67-S77.

4. Parkinson RW, Kay PR, Rawal A. A case for one-stage revision in infected total knee arthroplasty? The Knee. 2011;18(1):1-4.

5. Silva M, Tharani R, Schmalzried TP. Results of direct exchange or debridement of the infected total knee arthroplasty. Clin Orthop. 2002;404:125-131.

6. Masters JP, Smith NA, Foguet P, et al. A systematic review of the evidence for single stage and two stage revision of infected knee replacement. BMC Musculoskelet Disord. 2013;14:222.
7. Pelt CE, Grijalva R, Anderson L, et al. Two-stage revision TKA is associated with high complication and failure rates. Advances in Orthopedics. 2014;2014;7

8. Mortazavi SMJ, Vegari D, Ho A, et al. Two-stage exchange arthroplasty for infected total knee arthroplasty: predictors of failure. Clin Orthop. 2011;469(11):3049-3054.

9. Gulhane S, Vanhegan IS, Haddad FS. Single stage revision: regaining momentum. J Bone Joint Surg Br. 2012;94(11 Suppl A):120-122.

10. Berend KR, Lombardi AV, Morris MJ, et al. Two-stage treatment of hip periprosthetic joint infection is associated with a high rate of infection control but high mortality. Clin Orthop. 2013;471(2):510-518.

11. Freeman MA, Sudlow RA, Casewell MW, et al. The management of infected total knee replacements. Bone Jt J. 1985;67(5):764-768.

12. Gehrke T, Alijanipour P, Parvizi J. The management of an infected total knee arthroplasty. Bone Jt J. 2015;97-B(10 Suppl A):20-29.

13. Bernard L, Hoffmeyer P, Assal M, et al. Trends in the treatment of orthopaedic prosthetic infections. $J$ Antimicrob Chemother. 2004;53(2):127-129.

14. Chew E, Khan WS, Agarwal S, et al. Single stage knee arthroplasty revision surgery: a systematic review of the literature. Open Orthop $J$. 2015;9:504-510.

15. Zahar A, Kendoff DO, Klatte TO, et al. Can good infection control be obtained in one-stage exchange of the infected TKA to a rotating hinge design? 10-year results. Clin Orthop. 2016;474(1):81-87.

16. George DA, Konan S, Haddad FS. Single-stage hip and knee exchange for periprosthetic joint infection. $J$ Arthroplasty. 2015;30(12):2264 2270.

17. Haddad FS, Sukeik M, Alazzawi S. Is single-stage revision according to a strict protocol effective in treatment of chronic knee arthroplasty infections? Clin Orthop. 2015;473(1):8-14.

18. Singer J, Merz A, Frommelt L, et al. High rate of infection control with one-stage revision of septic knee prostheses excluding MRSA and MRSE. Clin Orthop. 2012;470(5):1461-1471.

19. Bauer T, Piriou P, Lhotellier L, et al. Results of reimplantation for infected total knee arthroplasty:107 cases. Rev Chir Orthop Reparatrice Appar Mot. 2006;92(7):692-700.

20. Jenny J-Y, Barbe B, Gaudias J, et al. High infection control rate and function after routine one-stage exchange for chronically infected TKA. Clin Orthop. 2013;471(1):238-243.

21. Klatte TO, Kendoff D, Kamath AF, et al. Single-stage revision for fungal peri-prosthetic joint infection: a single-centre experience. Bone $J t J$ 2014;96-B(4):492-496.

22. Kunutsor SK, Whitehouse MR, Lenguerrand E, et al. Re-infection outcomes following one- and two-stage surgical revision of infected knee prosthesis: a systematic review and meta-analysis. PLOS ONE. 2016;11(3):e0151537.

23. Tibrewal S, Malagelada F, Jeyaseelan L, et al. Single-stage revision for the infected total knee replacement: results from a single centre. Bone $J t$ J. 2014;96-B(6):759-764.

24. Whiteside LA, Peppers M, Nayfeh TA, et al. Methicillin-resistant Staphylococcus aureus in TKA treated with revision and direct intraarticular antibiotic infusion. Clin Orthop Relat Res.. 2011;469(1):26-33.

25. Whiteside LA, Roy ME. One-stage revision with catheter infusion of intraarticular antibiotics successfully treats infected THA. Clin Orthop. 2017;475(2):419-429. 
26. Li HK, Scarborough M, Zambellas R, et al. Oral versus intravenous antibiotic treatment for bone and joint infections (OVIVA): study protocol for a randomised controlled trial. Trials. 2015;16:583

27. One stage versus two stage for periprosthetic hip and knee infection - full text view. 2017.

28. von Foerster G, Klüber D, Käbler U. [Mid- to long-term results after treatment of 118 cases of periprosthetic infections after knee joint replacement using one-stage exchange surgery]. Orthopade. 1991;20(3):244-252.

29. Göksan SB, Freeman MA. One-stage reimplantation for infected total knee arthroplasty. J Bone Joint Surg Br. 1992;74(1):78-82.
30. Lu H, Kou B, Lin J. [One-stage reimplantation for the salvage of total knee arthroplasty complicated by infection]. Zhonghua Wai Ke Za Zhi. 1997;35(8):456-458.

31. Buechel FF, Femino FP, D'Alessio J. Primary exchange revision arthroplasty for infected total knee replacement: a long-term study. Am J Orthop Belle Mead NJ. 2004;33(4):190-198.

32. Sofer D, Regenbrecht B, Pfeil J. Early results of one-stage septic revision arthroplasties with antibiotic-laden cement. A clinical and statistical analysis. Orthopade. 2005;34(6):592-602. 